\title{
Enhancement of Ferroelectric Polarization Stability by Interface Engineering
}

\author{
H. Lu, X. Liu, J. D. Burton, C.-W. Bark, Y. Wang, Y. Zhang, D. J. Kim, A. Stamm, \\ P. Lukashev, D. A. Felker, C. M. Folkman, P. Gao, M. S. Rzchowski, X. Q. Pan, \\ C.-B. Eom, E. Y. Tsymbal, and A. Gruverman*
}

Switchable ferroelectric polarization in ultrathin-film heterostructures offers a promising functional basis for novel nanoelectronic devices. The main challenge for the implementation of such devices, however, is to overcome deleterious effects rendering ferroelectricity unstable, especially interface effects that become increasingly important as films are made thinner. Here, we demonstrate a synergetic experimental/theoretical approach to stabilize that ferroelectric polarization in $\mathrm{nm}$-thick ferroelectric films by interface engineering. We find that in the epitaxial $\mathrm{BaTiO}_{3}$ films sandwiched between $\mathrm{SrRuO}_{3}$ layers the $\mathrm{RuO}_{2} / \mathrm{BaO}$ interface termination sequence, which commonly occurs during unit-cell-by-unit-cell growth, is detrimental to ferroelectric stability due to the presence of a pinned interface dipole. Our first-principles and phenomenological modeling predict and experimental measurements confirm that switchable ferroelectric polarization can be achieved by depositing a very thin layer of $\mathrm{SrTiO}_{3}$ at the top interface to eliminate the unfavorable interface termination. These results indicate that interface engineering is a viable approach to stabilize ferroelectric polarization in ultrathin-film heterostructures.

Ferroelectrics are promising materials for a variety of technological applications such as non-volatile random access memories and high-density data storage devices. ${ }^{[1]}$ Continuing demand for further miniaturization of electronic devices brings up a problem of ferroelectric polarization stability at the

Dr. H. Lu, X. Liu, Dr. J. D. Burton, Y. Wang, Dr. D. J. Kim, A. Stamm,

Dr. P. Lukashev, Prof. E. Y. Tsymbal, Prof. A. Gruverman

Department of Physics and Astronomy

Nebraska Center for Materials and Nanoscience

University of Nebraska

Lincoln, NE 68588, USA

E-mail: agruverman2@unl.edu

Dr. C.-W. Bark, C. M. Folkman

Department of Materials Science and Engineering

University of Wisconsin-Madison

WI 53706, USA

Y. Zhang, P. Gao, Prof. X. Q. Pan

Department of Materials Science and Engineering

University of Michigan

Ann Arbor, MI 48109, USA

D. A. Felker, Prof. M. S. Rzchowski, Prof. C.-B. Eom

Department of Physics

University of Wisconsin-Madison

WI 53706, USA

DOI: 10.1002/adma.201104398 nanometer scale. ${ }^{[2,3]}$ In particular, one of the promising research directions on ferroelectric tunnel junctions ${ }^{[4-6]}$ relies entirely on the existence of stable polarization states in the ferroelectric barrier layers with thickness of just several unit cells. Switchable polarization of the barrier allows for a giant change in resistance in such junctions, known as the tunneling electroresistance effect, ${ }^{[7,8]}$ and control of the transport spin polarization if the electrodes are ferromagnetic. ${ }^{\left[{ }^{9-11]}\right.}$ Thus, maintaining and controlling a stable and switchable electric polarization in ferroelectric thin films down to the nanometer thickness range at room temperature is essential for exploiting the functionality of these materials for nanoelectronics applications.

Ferroelectric stability in thin films is largely determined by minimization of depolarizing field energy. The depolarizing field can be reduced by formation of screening charges at the film-electrode interfaces ${ }^{[12-14]}$ or by forming a periodical stripe domain structure. ${ }^{[15-19]}$ Although a free-electron charge in the metal electrodes is believed to provide the most efficient screening stabilizing the ferroelectric state down to nanometer dimensions, ${ }^{[12]}$ stable ferroelectricity has been also observed in nm-thick films without top electrodes..$^{[7,8,13,14]}$ In this case, charge compensation at the free polar surfaces can be provided by ionic adsorbates, as has also been inferred from electrostatic force microscopy measurements ${ }^{[20]}$ and first-principles calculations. ${ }^{[14]}$

The presence of two electrodes is essential for the functional control and reproducibility of ferroelectric-based device characteristics. However, in some cases the screening provided by conductive electrodes appears to be less effective than screening by adsorbates on free surfaces exposed to air. ${ }^{[8]}$ For example, deposition of the top $\mathrm{SrRuO}_{3}$ electrodes on $\mathrm{BaTiO}_{3}$ films results in severe relaxation to zero net polarization. ${ }^{[19,21]}$ This effect is a consequence of strong effective depolarizing fields due to incomplete screening or reduced interface capacitance effect. In addition, the presence of asymmetric interfaces may lead to a built-in electric field, whose direction is independent of polarization orientation resulting in two non-equivalent polarization states. ${ }^{[22]}$ In fact, such asymmetry may even destroy the stability of one of the polarization states, making the system only monostable in zero applied field and, therefore, non-ferroelectric. Addressing these detrimental effects is critical both for the fundamental understanding of the ferroelectric behavior at the nanoscale and related device performance.

One of the approaches to stabilize and even enhance ferroelectricity in nm-thick films has been offered by first-principles calculations suggesting that interface atomic structure and 
chemical bonding may significantly impact a ferroelectric state. ${ }^{[23,24]}$ In particular, interface engineering may be employed to eliminate the formation of a non-switchable interface dipole layer that is detrimental to ferroelectricity. In this paper, we exploit this approach to enhance ferroelectricity in thin $\mathrm{BaTiO}_{3}$ films sandwiched between two $\mathrm{SrRuO}_{3}$ electrodes. Using firstprinciples calculations and experimental studies we demonstrate that introduction of a very thin layer of $\mathrm{SrTiO}_{3}$ at the $\mathrm{SrRuO}_{3} / \mathrm{BaTiO}_{3}$ interface eliminates the unfavorable $\mathrm{RuO}_{2} /$ $\mathrm{BaO}$ termination leading to a stable and switchable ferroelectric polarization.

Our first-principles calculations are performed using density functional theory as described in the Experimental Section. We consider systems where $\mathrm{SrRuO}_{3}$ electrodes are separated by an insulating layer made of either (i) $n$ unit cells (u.c.) of $\mathrm{BaTiO}_{3}$, or (ii) n-2 u.c. of $\mathrm{BaTiO}_{3}$ plus a 2-u.c.-thick layer of $\mathrm{SrTiO}_{3}$. Since both $\mathrm{SrRuO}_{3}$ and $\mathrm{BaTiO}_{3}$ belong to the same pseudocubic perovskite-oxide family of structures with formula $\mathrm{ABO}_{3}$, we consider a $1 \times 1$ supercell stacked along the [001] pseudocubic direction using the typical $A O-B \mathrm{O}_{2}$ stacking of the atomic layers, where $A=\mathrm{Ba}$ or $\mathrm{Sr}$ and $B=\mathrm{Ti}$ or Ru. The in-plane lattice constant is constrained to $a=3.87 \AA$ which is the calculated LDA lattice constant of cubic $\mathrm{SrTiO}_{3}$. This constraint simulates the epitaxial strain of coherent growth on a single crystal $\mathrm{SrTiO}_{3}$ substrate, just as in our experimentally grown samples. This inplane constraint imposes a compressive strain on the $\mathrm{BaTiO}_{3}$, yielding a $c / a$ ratio of 1.055 , ensuring a polarization along the [001] direction.

When $\mathrm{BaTiO}_{3}$ is deposited in the [001] direction by pulsed laser deposition (PLD) it grows unit-cell-by-unit-cell. This results in a stoichiometric film with different atomic terminations on the two sides, i.e. one surface/interface will be $\mathrm{BaO}$ terminated while the other will be $\mathrm{TiO}_{2}$ terminated. Therefore, since the usual $A O-B \mathrm{O}_{2}$ stacking sequence is preserved at the interfaces in a heterostructure with $\mathrm{SrRuO}_{3}$ electrodes, the bottom interface has the $\mathrm{TiO}_{2}$-SrO termination sequence and the top interface has the $\mathrm{RuO}_{2}-\mathrm{BaO}$ termination sequence. The structures of these asymmetric interface terminations are schematically shown in Figure 1. In this representation, for comparison with our experimental results below, the termination on the left side corresponds to the bottom interface while the top interface is on the right side.

Ferroelectricity in $\mathrm{BaTiO}_{3}$ manifests itself as a symmetrybreaking structural distortion away from the perfect cubic perovskite structure. The distortion consists of relative displacement along one of the 6 cubic axes of the $\mathrm{Ti}$ and $\mathrm{Ba}$ cations from their co-planar $\mathrm{O}$ anions in each $\mathrm{TiO}_{2}$ and $\mathrm{BaO}$ atomic plane, respectively. In the case of bi-axial compressive-straining (a) $\odot$ Sr $\diamond \mathrm{Ba} \diamond \mathrm{Ru} \diamond \mathrm{Ti} \circ \mathrm{O}$

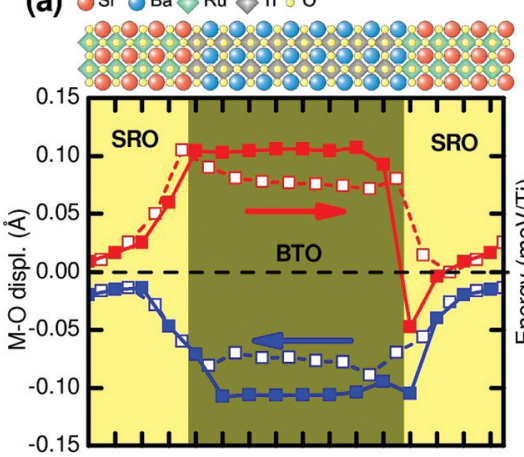

(d)

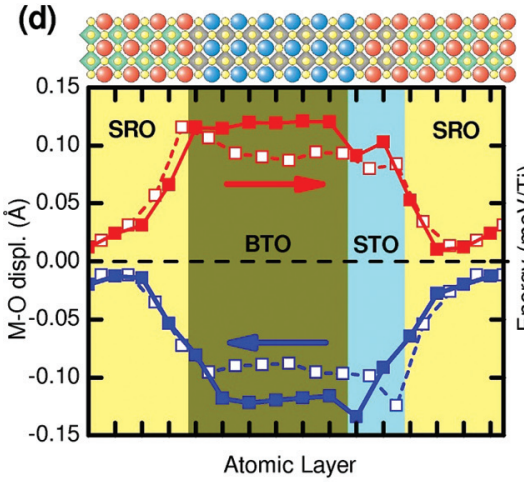

(b)

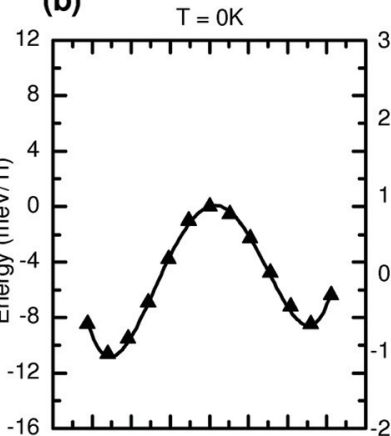

(c)

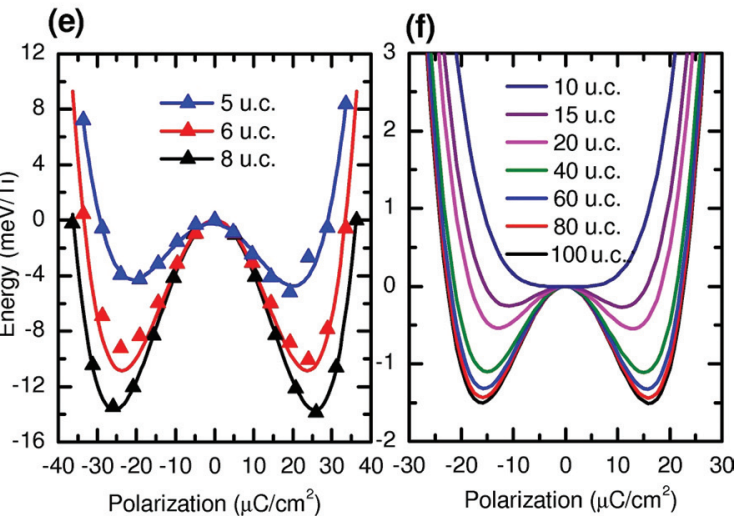

(e)

Figure 1. (a) Schematic side view of the layer-by-layer atomic positions the $\mathrm{SrRuO}_{3} /\left(\mathrm{BaTiO}_{3}\right)_{8} / \mathrm{SrRuO}_{3}$ structure and profile of the relative metal-oxygen $(\mathrm{M}-\mathrm{O})$ displacements in each atomic layer of the $\mathrm{SrRuO}_{3} /\left(\mathrm{BaTiO}_{3}\right)_{8} / \mathrm{SrRuO}_{3}$ structure. The solid symbols are $B-\mathrm{O}_{2}$ displacements $(B=\mathrm{Ru}$ or Ti) and the open symbols are $A-O$ displacements $(A=\mathrm{Sr}$ or $\mathrm{Ba})$. Red and blue curves correspond to the up and down polarization states, respectively. (b) Total energy per Ti atom calculated from first-principles (symbols) and from the phenomenological model (a curve) as a function of average polarization for $\mathrm{SrRuO}_{3} /\left(\mathrm{BaTiO}_{3}\right)_{n} / \mathrm{SrRuO}_{3}$ heterostructures with $n=8$. (c) Free energy per Ti atom at $T=300 \mathrm{~K}$ as a function of average polarization calculated using the phenomenological model for (a) the $\mathrm{SrRuO}_{3} /\left(\mathrm{BaTiO}_{3}\right)_{n} / \mathrm{SrRuO}_{3}$ structures. (d-f) Same as for (a-c) except for SrRuO${ }_{3} /\left(\mathrm{BaTiO}_{3}\right)_{n-2} /\left(\mathrm{SrTiO}_{3}\right)_{2} /$ $\mathrm{SrRuO}_{3}$ structures and $n=5,6$, and 8 . 
of bulk $\mathrm{BaTiO}_{3}$ the tetragonal $c$-axis is the favored axis for the distortions and the two corresponding polarization directions are degenerate in energy. In the heterostructures we consider, however, the asymmetric interfaces break this degeneracy and the two polarization directions become inequivalent, as is evident from the calculated structural distortions for the two polarization states in the 8-u.c. system (Figure 1a). We see that in addition to the reversible distortions in the $\mathrm{BaTiO}_{3}$ layer there are polar distortions that penetrate into the $\mathrm{SrRuO}_{3}$ electrode, in agreement with the previous calculations. ${ }^{[25]}$ On the bottom interface (on the left in Figure 1a), terminated by $\mathrm{TiO}_{2}-\mathrm{SrO}$, these polar distortions in $\mathrm{SrRuO}_{3}$ follow the polarization direction in the $\mathrm{BaTiO}_{3}$, consistent with the penetration of a screened electric field into the metallic $\mathrm{SrRuO}_{3}$. However, on the top interface (on the right in Figure 1a) terminated by $\mathrm{RuO}_{2}-\mathrm{BaO}$ we find that the polar displacement direction in the first unitcell of $\mathrm{SrRuO}_{3}$ does not follow the polarization of the $\mathrm{BaTiO}_{3}$. Most notably the first $\mathrm{RuO}_{2}$ layer exhibits a significant negative polar displacement whose magnitude remains appreciable even when the polarization of the $\mathrm{BaTiO}_{3}$ opposes it.

The interface dipole arises because of a mismatch between ionic radii: the Sr-Ti interface can be viewed as one cell of $\mathrm{SrTiO}_{3}$, whose cationic radii complement one another so that it has a preference to be centrosymmetric, and will therefore only develop off-centering in response to an electric field. At the $\mathrm{Ba}-\mathrm{Ru}$ interface, however, $\mathrm{Ba}$ has a larger ionic radius than $\mathrm{Sr}$ and, just as it does in $\mathrm{BaTiO}_{3}$, leads to off-centering of the B-site cation $\left(\mathrm{Ru}^{4+}\right.$ in this case) with respect to the co-planar oxygen ions. The asymmetric environment of the interfacial $\mathrm{Ru}$, however, strongly favors only one orientation (negative in our case). In addition, $\mathrm{BaRuO}_{3}$, which is essentially what we have at the Ba-Ru interface, only assumes the pseudo-cubicperovskite structure similar to $\mathrm{SrRuO}_{3}$ at high pressures due to the larger A-site cationic radius. ${ }^{[26]}$ This is a different origin of fixed interface displacements than the charge mismatch effect recently found in Ref.[27] Below, we show that this pinned interface dipole plays a detrimental role in the stability of a switchable ferroelectric polarization.

The effect of asymmetry on stability has been further studied by examining the energy profile that establishes the two states. We do this by taking the fully relaxed polarization structures, which differ only in their atomic $z$ coordinates, and construct a series of structures which interpolate between these two stable states, calculating the total energy for each such structure. In addition to the total energy we calculate the average polarization, $P$, in the spacer using the approach developed in Ref.[25] based on the Ginzburg-Landau-Devonshire theory applied to a ferroelectric film. ${ }^{[28]}$ By fitting the first-principles calculations for $n=$ 8 structures we find an expression for the free energy, which can be easily adapted to study different thicknesses and even finite temperatures (further details can be found elsewhere ${ }^{[29]}$ ).

In Figure 1b, we plot the double well profile for $n=8$ unit cells of $\mathrm{BaTiO}_{3}$ derived from the structures shown in Figure 1a. The phenomenological fit (curve) to the first-principles data (points) is clearly very good. We see that the positive polarization state is higher in energy, and has a smaller effective barrier height for reversal, than does the negative polarization state. This asymmetry in the energy profile is a natural consequence of the downward pointing pinned interface dipole at the top,
$\mathrm{RuO}_{2}-\mathrm{BaO}$ terminated, interface: the dipole partially cancels depolarization effects for the down polarization state, whereas the dipole adds to the already unfavorable depolarization effects in the up polarization state. Since this is an interface effect we can expect the influence of the pinned dipole to increase for thinner ferroelectric spacer layers. To explore this, we additionally performed relaxation calculations for heterostructures with $n=4,5$ and 6 unit cells of $\mathrm{BaTiO}_{3}$. Surprisingly, we find that it was possible only to stabilize the downward polarization state in each of these thinner structures: all attempts at finding an upward polarization state result in relaxation back to the downward polarization state. This indicates that all upward polarization states are energetically unstable, which is consistent with the strong dipole at the $\mathrm{RuO}_{2} / \mathrm{BaO}$ interface.

The detrimental effect of the $\mathrm{RuO}_{2}-\mathrm{BaO}$ interface termination can be alleviated by replacing two $\mathrm{BaTiO}_{3}$ unit cells at the top interface with $\mathrm{SrTiO}_{3}$ (Figure 1d). This will make the interface termination sequence with the top electrode $\mathrm{RuO}_{2}-\mathrm{SrO}$, or equivalently $\mathrm{SrO}-\mathrm{TiO}_{2}$ just as it is with the bottom interface. Fully relaxed calculations of the $\mathrm{SrRuO}_{3} /\left(\mathrm{BaTiO}_{3}\right)_{6}\left(\mathrm{SrTiO}_{3}\right)_{2} /$ $\mathrm{SrRuO}_{3}$ heterostructure reveal that both states are stable and the polar displacements throughout the heterostructure, even those in the $\mathrm{SrRuO}_{3}$, follow the polarization direction in the $\mathrm{BaTiO}_{3}$ (Figure 1d). Using the same procedure as before we calculate the double-well energy profile for structures containing 2 unit cells of $\mathrm{SrTiO}_{3}$ and 3, 4 and 6 unit cells of $\mathrm{BaTiO}_{3}$. Remarkably, in contrast to the pure $\mathrm{BaTiO}_{3}$ structures, full relaxation calculations establish two stable polarization states in all cases (Figure 1e). Our phenomenological model matches quite well with the zero-temperature first-principles data for all interface-engineered heterostructures (solid lines in Figure 1e).

In addition to the removal of the unfavorable asymmetry induced by the interface dipole, the insertion of the $\mathrm{SrTiO}_{3}$ also leads to a further enhancement of the ferroelectric stability, as can be seen by comparing the energy wells for the $n=8$ structures in Figure 1(b) and (e). The well depth (effective barrier height) of the downward polarization state in the structure with the $\mathrm{SrTiO}_{3}$ interface layer is $\sim 4 \mathrm{meV} / \mathrm{Ti}$ deeper (larger) than for the pure $\mathrm{BaTiO}_{3}$ heterostructure. This can come from the enhanced screening of the depolarizing field in the $\mathrm{BaTiO}_{3}$ due to the large dielectric response of $\mathrm{SrTiO}_{3}$.

In addition to the zero-temperature results, the phenomenological model has been extrapolated to room temperature by assuming a simple temperature dependence of the free energy coefficients. ${ }^{[29]}$ We find that at room temperature a ferroelectric monodomain state in a $\mathrm{SrRuO}_{3} / \mathrm{BaTiO}_{3} / \mathrm{SrRuO}_{3}$ heterostructures can be stabilized only above $\sim 20$ u.c. (Figure $1 \mathrm{c}$ ). This is in contrast to a $\mathrm{SrRuO}_{3} /\left(\mathrm{BaTiO}_{3}\right)_{6}\left(\mathrm{SrTiO}_{3}\right)_{2} / \mathrm{SrRuO}_{3}$ heterostructure, where the model predicts that the unfavorable contribution from the interface dipole on ferroelectric stability is alleviated at room temperature even for 15 u.c. of $\mathrm{BaTiO}_{3}$, and possibly thinner (Figure 1f).

To verify these theoretical predictions we have grown several epitaxial $\mathrm{BaTiO}_{3}$-based heterostructures with engineered interfaces (Figure 2) and studied their switching behavior. Single-crystalline $\mathrm{BaTiO}_{3}$ films (with a thickness of 24 unit cells) have been fabricated by pulsed laser deposition using atomically-controlled layer-by-layer growth on atomically smooth (001) $\mathrm{SrTiO}_{3}$ substrates with single-crystalline top and 
(a)

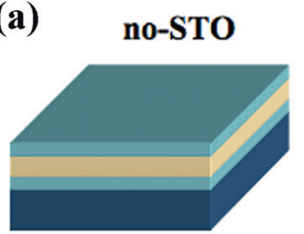

$\mathrm{SrRuO}_{3}$

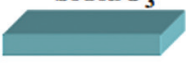

(b)

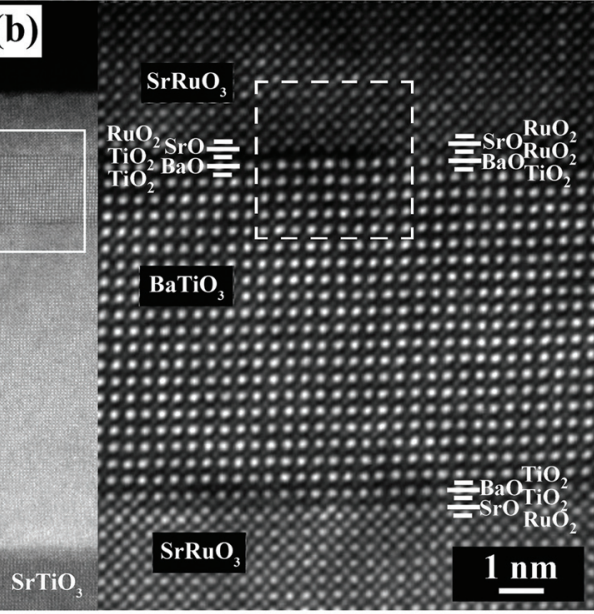

(d)

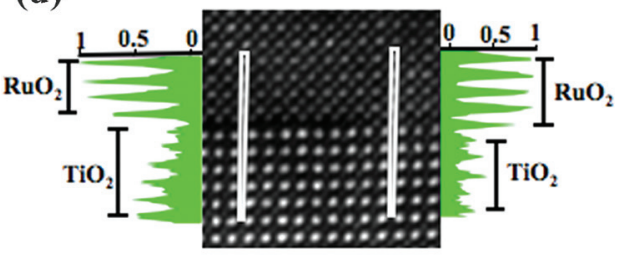

(f)

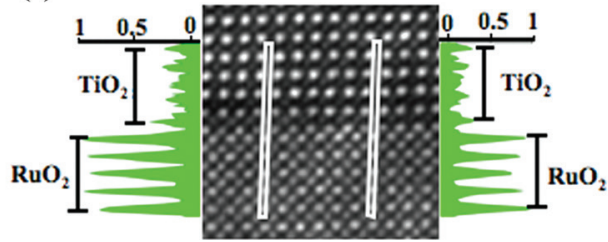

t-STO

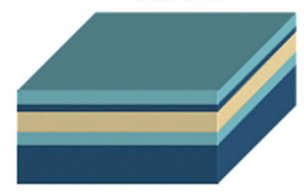

$\mathrm{BaTiO}_{3}$
b-STO

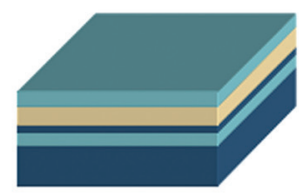

$\mathrm{SrTiO}_{3}$

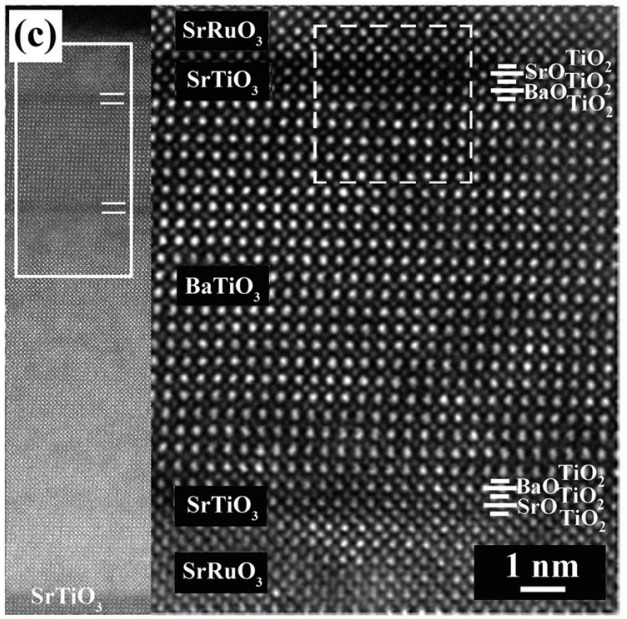

(e)

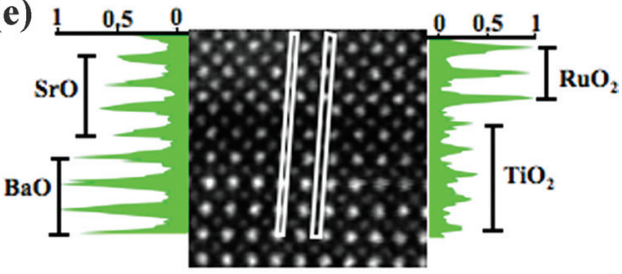

(g)

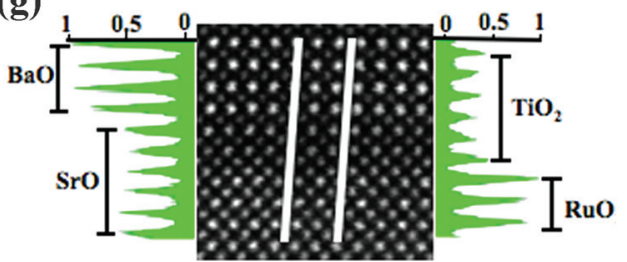

Figure 2. (a) Schematic diagrams of the reference $\mathrm{SrRuO}_{3} / \mathrm{BaTiO}_{3} / \mathrm{SrRuO}_{3}$ heterostructure (no-STO) and heterostructures with engineered top (t-STO) and bottom (b-STO) interfaces. Thickness of the $\mathrm{BaTiO}_{3}$ and $\mathrm{SrTiO}_{3}$ layers is 24 u.c. and 2 u.c., respectively. (b) STEM image of the reference $\mathrm{SrRuO}{ }_{3} /$ $\mathrm{BaTiO}_{3} / \mathrm{SrRuO}_{3}$ heterostructure. A white block in the inset on the left indicates an area shown at higher magnification on the right. (c) STEM image of the $\mathrm{SrRuO}_{3} / \mathrm{SrTiO}_{3} / \mathrm{BaTiO}_{3} / \mathrm{SrTiO}_{3} / \mathrm{SrRuO}_{3}$ heterostructure. A white block in the inset on the left indicates an area shown at higher magnification on the right. Note the same terminations at the top and bottom $\mathrm{BaTiO}_{3} / \mathrm{SrTiO}_{3}$ interfaces. (d) The intensity profiles of the top interface of the reference $\mathrm{SrRuO}_{3} / \mathrm{BaTiO}_{3} / \mathrm{SrRuO}_{3}$ heterostructure indicating a mixture of $\mathrm{RuO}_{2}-\mathrm{BaO}$ and $\mathrm{TiO}_{2}-\mathrm{SrO}$ terminations (also shown in 2 (b)). (e) The intensity profiles showing the $\mathrm{TiO}_{2}-\mathrm{BaO}$ termination at the top interface of the $\mathrm{SrRuO}_{3} / \mathrm{SrTiO}_{3} / \mathrm{BaTiO}_{3} / \mathrm{SrTiO}_{3} / \mathrm{SrRuO}_{3}$ heterostructure. (f) The intensity profiles of the bottom interface of the reference heterostructure indicating termination of the $\mathrm{TiO}_{2}$-SrO type (also shown in $2(\mathrm{~b})$ ). (g) The intensity profiles indicating the $\mathrm{TiO}_{2}-\mathrm{SrO}$ termination at the bottom interface of the $\mathrm{SrRuO}_{3} / \mathrm{SrTiO}_{3} / \mathrm{BaTiO}_{3} / \mathrm{SrTiO}_{3} / \mathrm{SrRuO}_{3}$ heterostructure.

bottom $\mathrm{SrRuO}_{3}$ electrodes. ${ }^{[30,31]}$ Control of epitaxial growth of the $\mathrm{BaTiO}_{3}$ films has been ensured by in situ monitoring of RHEED intensity oscillations and RHEED patterns.

As has been proposed in the modeling approach, interface engineering has been realized by introducing a $\mathrm{SrTiO}_{3}$ layer with the thickness of 2 unit cells between the $\mathrm{BaTiO}_{3}$ and $\mathrm{SrRuO}_{3}$ layers (Figure 2a). The atomic structure of interfaces was directly determined by spherical aberration (Cs) corrected scanning transmission electron microscopy (STEM). Crosssectional high-angle annular dark field (HAADF) image of the heterostructures without and with STO layers, viewed along [100] zone axis, are shown in Figure $2 \mathrm{~b}$ and $2 \mathrm{c}$, respectively. The high-resolution HAADF images revealed that the $\mathrm{BaTiO}_{3}$ and $\mathrm{SrRuO}_{3}$ layers being fully commensurate with the $\mathrm{SrTiO}_{3}$ substrate and showed well-defined interfaces (Figures $2 \mathrm{~b}$ and $2 \mathrm{c}$ ). The intensity profile of the top interface in $\mathrm{SrRuO}_{3} / \mathrm{BaTiO}_{3} /$ 
$\mathrm{SrRuO}_{3}$, referred to as the reference, or no-STO, heterostructure, reveals a mixture of $\mathrm{RuO}_{2}-\mathrm{BaO}$ and $\mathrm{TiO}_{2}-\mathrm{SrO}$ terminations (Figure 2d) while the bottom ferroelectric-electrode interface shows only $\mathrm{TiO}_{2}-\mathrm{SrO}$ termination (Figure 2f). Introduction of the $\mathrm{SrTiO}_{3}$ layer at the top interface changes its termination to the $\mathrm{TiO}_{2}-\mathrm{BaO}$ type (Figure 2e), while the $\mathrm{SrTiO}_{3}$ layer at the bottom interface leaves the $\mathrm{TiO}_{2}$-SrO type termination intact (Figure 2g). The corresponding $\mathrm{SrRuO}_{3} / \mathrm{SrTiO}_{3} / \mathrm{BaTiO}_{3} /$ $\mathrm{SrRuO}_{3}$ and $\mathrm{SrRuO}_{3} / \mathrm{BaTiO}_{3} / \mathrm{SrTiO}_{3} / \mathrm{SrRuO}_{3}$ heterostructures are referred to as t-STO and b-STO, respectively.

Ferroelectric properties have been characterized using polarization hysteresis and scanning probe microscopy (SPM) measurements. The hysteresis loops of the reference $\mathrm{SrRuO}_{3} / \mathrm{BaTiO}_{3} /$ $\mathrm{SrRuO}_{3}$ sample (Figure 3a) show almost a paraelectric-type behavior with the remanent polarization well below $1 \mu \mathrm{C} / \mathrm{cm}^{2}$ and a loop width of about $50 \mathrm{mV}$. To alleviate a strong nonlinear dielectric contribution effect and enable a more accurate measurement of such a low polarization value we applied a pulsed testing method. ${ }^{[32]}$ In $\mathrm{SrRuO}_{3} / \mathrm{BaTiO}_{3} / \mathrm{SrRuO}_{3}$, the remanent polarization $2 P_{r}$, defined from the difference between the switched $\mathrm{P}^{*}$ and nonswitched $P^{\wedge}$ polarization signals, shown in Figure $3 \mathrm{~b}$, is about $0.5 \mu \mathrm{C} / \mathrm{cm}^{2}$. (For comparison, the $2 P_{r}$ value of $100 \mu \mathrm{C} / \mathrm{cm}^{2}$ was reported for 200 -nm-thick $\mathrm{BaTiO}_{3}$ film on $\mathrm{GdScO}_{3}$ substrate with $\mathrm{SrRuO}_{3}$ top and bottom electrodes ${ }^{[30]}$ ). Changing the termination type at the top interface from $\mathrm{RuO}_{2}$ $\mathrm{BaO}$ to $\mathrm{TiO}_{2}-\mathrm{BaO}$ (t-STO sample) increases the remanent polarization by almost a factor of 3 to about $1.6 \mu \mathrm{C} / \mathrm{cm}^{2}$, which is consistent with the changes predicted by the first-principle modeling. On the other hand, the remanent polarization value detected in the b-STO sample is very similar to that in the reference sample. This similarity can be expected as the in this case the interface terminations are identical for both structures. Introduction of the $\mathrm{SrTiO}_{3}$ layer at both top and bottom interfaces produces the same effect as modification of only the top interface (see Figure S1 in supplementary section). This finding confirms that the main mechanism behind the polarization retention enhancement is the change in termination structure at the top interface.

While polarization hysteresis loop data are related to the net polarization, PFM provides information on the local (nanoscale) ferroelectric polarization. For the tetragonal (001)-oriented ferroelectrics as the $\mathrm{BaTiO}_{3}$ films used in our study, the PFM amplitude signal is determined by the $\mathrm{d}_{33}$ piezoelectric coefficient which is proportional to the normal component of the local polarization: $d_{33} \sim e e_{0} P \cdot{ }^{[33]}$ Ramping the poling dc voltage and monitoring the accompanying change in the PFM amplitude signal allows testing the switching behavior at the spatial range determined by the tip-sample contact area (which is on the order of $10 \mathrm{~nm}$ in diameter). Local PFM switching measurements reveal a typical hysteretic polarization behavior for all the heterostructures (Figure 4a). Before the measurements, the samples were poled by application of voltage pulses of $+4 \mathrm{~V}$, which is well above the threshold voltage. In PFM testing, the parameter directly related to the remanent polarization value is the initial piezoresponse amplitude signal at $0 \mathrm{~V}$. It can be seen that for the reference $\mathrm{SrRuO}_{3} / \mathrm{BaTiO}_{3} / \mathrm{SrRuO}_{3}$ and for the b-STO samples, the initial PFM amplitude and thus the local remanent polarization values are close to zero, which is consistent with zero net polarization revealed by $P-V$ hysteresis (a)

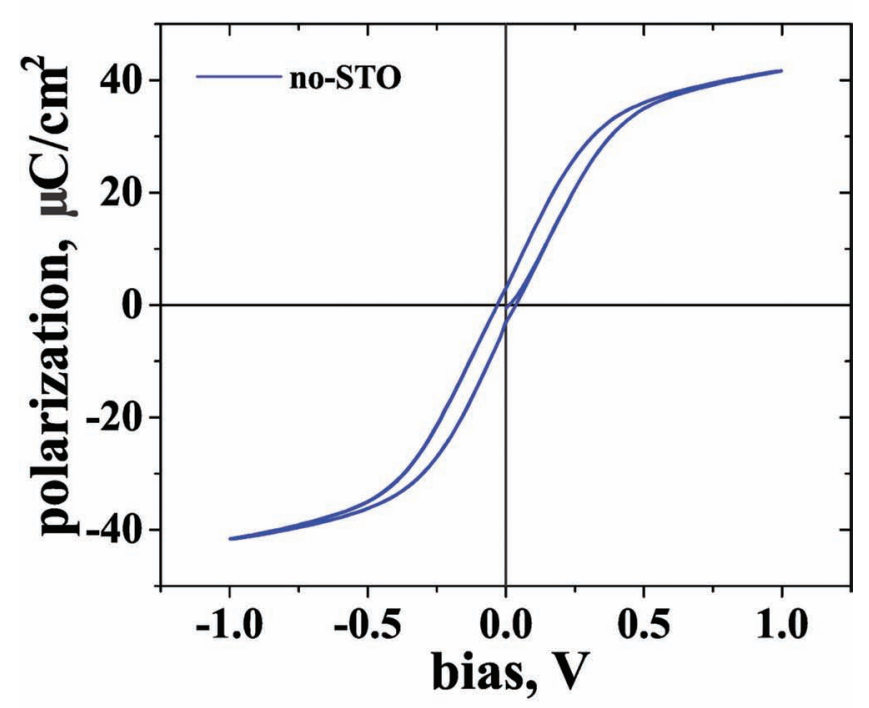

(b)

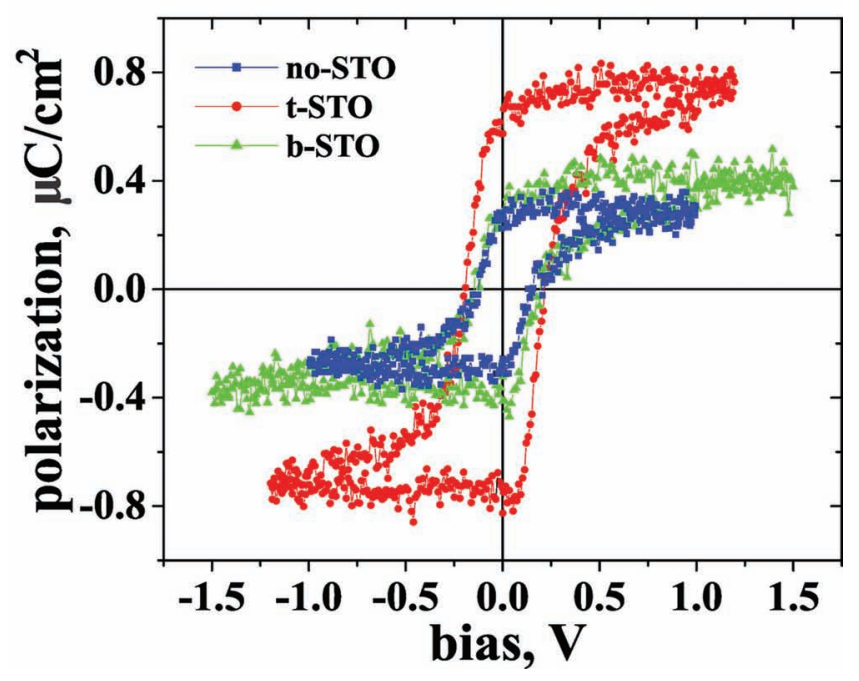

Figure 3. (a) Conventional and (b) remanent $P-V$ hysteresis loops measured by pulsed testing in no-STO, t-STO and b-STO heterostructures. The loops in (a) exhibit a width of $50 \mathrm{mV}$ and low remanent polarization indicating an unstable polarization state. Note an almost $200 \%$ increase in the remanent polarization for the t-STO sample in comparison with the no-STO and b-STO samples.

measurements. On the other hand, the t-STO sample exhibits a much higher initial PFM amplitude signal illustrating an enhanced remanent polarization in the sample with the engineered top interface. This effect was also observed when samples were poled by the opposite voltage (not shown here).

To obtain a nanoscopic insight into the domain structure associated with the observed retention behavior, we employed a PFM imaging approach. Given that the $\mathrm{BaTiO}_{3}$ films used in the present study are fully coherent with the $\mathrm{SrTiO}_{3}$ substrates, which impose compressive stress, it is expected that the films would be in the polar state and only $180^{\circ}$ domains would be 
(a)

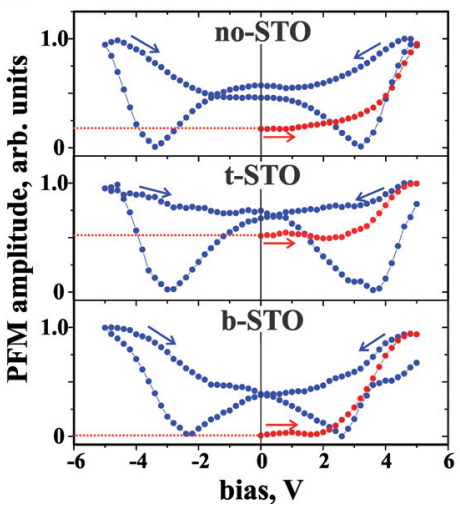

(b)

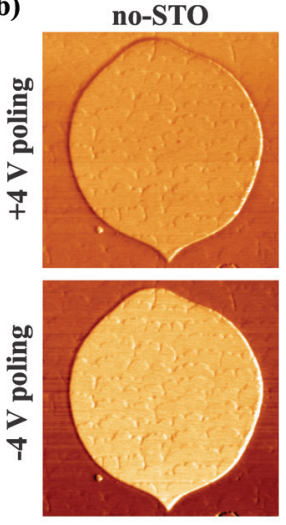

t-STO

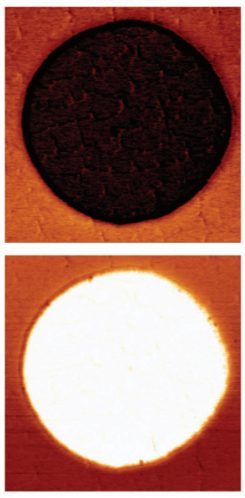

b-STO

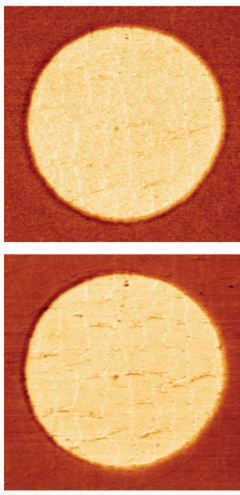

Figure 4. (a) PFM hysteresis loops for no-STO, t-STO and b-STO heterostructures after application of $+4 \mathrm{~V}$ poling pulses. The first quarters of the cycles are shown in red with arrows indicating the direction of voltage change. A difference in the initial (at zero bias) PFM amplitude illustrates a difference in remanent polarization values for different heterostructures. (b) EFM images of the no-STO, t-STO and b-STO heterostructures after poling by $+/-4 \mathrm{~V}$ pulses. Contrast inversion upon the change of the pulse polarity is attributed to the change of screening charge on the electrodes and indicates a presence of a switchable polarization with two stable states.

present. It is also expected that to minimize the depolarizing energy, the system could break up into a system of $180^{\circ}$ periodic antiparallel domains of equal volume fraction thus bringing the net polarization to zero. However, PFM imaging of the reference $\mathrm{SrRuO}_{3} / \mathrm{BaTiO}_{3} / \mathrm{SrRuO}_{3}$ structure, which should be able to reveal domains as small as $10 \mathrm{~nm}$ in size, ${ }^{[34]}$ did not show any signature of the domain structure neither in the as-grown heterostructures nor after poling. This failure to observe domains by PFM can be explained if one recalls that the direct experimental measurements of ultrathin $\mathrm{BaTiO}_{3}$ films by Raman spectroscopy yielded a domain period value of $6.3 \mathrm{~nm},{ }^{[35]}$ which is below the PFM spatial resolution limit.

Nevertheless, the SPM approach still can offer additional support to the proposed mechanism of polarization retention enhancement. We have undertaken Electrostatic Force Microscopy (EFM) studies of the $\mathrm{BaTiO}_{3}$ heterostructures subjected to poling by positive and negative voltage pulses. Note that EFM is sensitive to the presence of the surface charge and, as such, can address the issue of polarization stability by detecting the screening charge retained on the electrodes after poling. The EFM images of the reference sample and samples with engineered heterostructures after $+/-4 \mathrm{~V}$ poling are shown in Figure 3b. It can be seen that while poling of the reference $\mathrm{SrRuO}_{3} / \mathrm{BaTiO}_{3} / \mathrm{SrRuO}_{3}$ sample by negative or positive pulses does not lead to any contrast change, the same poling procedure performed on the t-STO sample results in the appearance of strong reversible EFM contrast suggesting induction of the screening charge in the electrode. For the experimental conditions used in these measurements, dark EFM contrast corresponds to the positive charge signal (respectively, bright contrast indicates negative charges). Based on this observation of the reversible EFM signal and its correlation with the sign of the poling voltage it can be confirmed that the resulting EFM contrast is indeed due to the screening charge on the electrodes and thus indicates a presence of stable polarization in the t-STO sample after poling. ${ }^{[36]}$ Similarly, contrast inversion has been observed in the sample where both interfaces have been engineered (Figure S2). On the other hand, there is no change in the EFM signal after poling of the b-STO sample indicating zero net polarization. This effect is consistent with experimental results above showing that it is engineering of the top interface only that brings about polarization retention enhancement.

As a final note, it should be mentioned that the theoretically predicted effect of the interface engineering on polarization enhancement is only in qualitative agreement with the experiment. Interface engineering, while enhancing the remanent polarization by a factor of 3 , did not lead to full recovery of polarization to its bulk value. This result illustrates a significant role of other effects and parameters in minimizing the depolarizing energy, such as polarization electronic screening and possible defect formation at the interfaces. In addition, stability enhancement by interface engineering in the first-principle calculations is based on eliminating an ideal uniform $\mathrm{RuO}_{2}$ / $\mathrm{BaO}$ termination at the top interface, whereas our experimental STEM data shows that the top interface of our $\mathrm{SrRuO}_{3} /$ $\mathrm{BaTiO}_{3} / \mathrm{SrRuO}_{3}$ sample exhibits a mixture of different interface terminations.

In conclusion, a detrimental effect on polarization retention of the $\mathrm{RuO}_{2} / \mathrm{BaO}$ interface termination sequence in ultrathin epitaxial $\mathrm{SrRuO}_{3} / \mathrm{BaTiO}_{3} / \mathrm{SrRuO}_{3}$ heterostructures has been predicted by first-principles calculations. It has been suggested that significant retention enhancement can be achieved by depositing a very thin layer of $\mathrm{SrTiO}_{3}$ at the interface to eliminate the unfavorable interface termination. Validity of the proposed approach to polarization stabilization has been confirmed by experimental measurements of polarization retention behavior in a set of model heterostructures with atomically engineered interfaces. The obtained results indicate that interface engineering is a viable approach to enhancement polarization retention in ultrathin ferroelectric heterostructures.

\section{Experimental Section}

Modeling: First-principles density functional calculations were performed using the Vienna Ab Initio Simulation Package (VASP). Exchange and correlation effects were accounted for using the local 
density approximation (LDA). Self-consistent calculations and atomic relaxations were performed using an energy cutoff of $500 \mathrm{eV}$ for the plane wave expansion of the wavefunctions and an $8 \times 8 \times 1$ MonkhorstPack k-point grid for sampling of the Brillouin zone. Atomic relaxations were performed until the Hellmann-Feynman force on each atom was less than $30 \mathrm{meV} / \AA$.

Materials: Single-crystalline epitaxial $\mathrm{SrRuO}_{3}(3 \mathrm{~nm}) / \mathrm{BaTiO}_{3}(24$ u.c. $) /$ $\mathrm{SrRuO}_{3}(30 \mathrm{~nm})$ heterostructures have been grown by pulsed laser deposition (PLD) on atomically smooth (001) $\mathrm{SrTiO}_{3}$ substrates. Reflection high-energy electron diffraction (RHEED) has been used for in-situ monitoring of the layer-by-layer growth process. Top $\mathrm{SrRuO}_{3}$ electrodes of about $6 \mu \mathrm{m}$ in diameter and $3 \mathrm{~nm}$ thick have been patterned by lift-off lithography. Before deposition, low angle miscut $\left(<0.1^{\circ}\right) \mathrm{SrTiO}_{3}$ substrates were etched using buffered HF acid for 90 seconds to maintain Ti-termination and then were annealed in oxygen at $1000{ }^{\circ} \mathrm{C}$ for 12 hours to create atomically smooth surfaces with single-unit-cell-height steps. During deposition of all the layers except for the top $\mathrm{SrRuO}_{3}$ electrode substrate temperature was maintained at $680^{\circ} \mathrm{C}$ with chamber oxygen pressure kept at 150 mTorr. The top $\mathrm{SrRuO}_{3}$ electrode was grown at lower temperature $\left(600^{\circ} \mathrm{C}\right)$ to avoid intermixing. The samples were annealed at growth temperature and $1 \mathrm{~atm}$ oxygen pressure for 30 minutes and then cooled down to room temperature. The resulting surface of the $\mathrm{BaTiO}_{3}$ thin films was atomically smooth with single unit cell height steps.

Experimental Measurements: Polarization imaging and local switching spectroscopy has been performed using a resonant-enhanced Piezoresponse Force Microscopy (MFP-3D, Asylum Research). Conductive silicon cantilevers (DPE18/Pt, Mikromasch) have been used in this study. PFM hysteresis loops were obtained at fixed locations on the top $\mathrm{SrRuO}_{3}$ electrodes as a function of a dc switching pulses $(25 \mathrm{~ms})$ with a superimposed ac modulation bias with amplitude of $0.8 \mathrm{~V}_{\mathrm{p}-\mathrm{p}}$ at $320 \mathrm{kHz}$. PFM local spectroscopy was carried out after application of a preset voltage pulse to measure initial response, which allows an indirect measurement of polarization state. Electrostatic force microscopy (EFM) imaging of the heterostructures has been performed at a resonance frequency of $65 \mathrm{kHz}$ with a tip under $+2 \mathrm{~V}$ dc bias.

Conventional and differential (remanent) $P-V$ hysteresis loops have been measured using Precision SC tester (Radiant Technologies) in the top-to-bottom contact configuration at the modulating frequency of $500 \mathrm{~Hz}$ with voltage sweeping from $-1.5 \mathrm{~V}$ to $1.5 \mathrm{~V}$. Electrical connection to the top $\mathrm{SrRuO}_{3}$ electrodes was realized via a conductive PFM probe, while the bottom electrode was grounded. Remanent polarization has been measured by using two sets of pulse trains (see supplementary part Figure S3).

The microstructural analysis has been carried out with a spherical aberration-corrected JEOL $2100 \mathrm{~F}$ scanning transmission electron microscope, operating at $200 \mathrm{kV}$. The intensity profiles have been measured using the Gatan DigitalMicrograph software.

\section{Supporting Information}

Supporting Information is available from the Wiley Online Library or from the author.

\section{Acknowledgements}

This research was supported by the National Science Foundation (NSF) through Materials Research Science and Engineering Center (NSF Grant No. 0820521) at University of Nebraska-Lincoln (UNL), by the U.S. Department of Energy, Office of Basic Energy Sciences, Division of Materials Sciences and Engineering (DOE grant DE-SC0004876) and by the Experimental Program to Stimulate Competitive Research (NSF Grant No. EPS-1010674). Computations were performed at the UNL Holland Computing Center. Work at the University of Michigan was supported by the U.S. Department of Energy under award DE-FG02-07ER46416 and by NSF under awards DMR-0907191, DMR-0820404 and DMR-0723032 (aberration-corrected TEM instrument).

Received: November 11, 2011

Revised: December 23, 2011

Published online: January 25, 2012

[1] J. F. Scott, Ferroelectric Memories, Springer, Germany 2000.

[2] T. M. Shaw, S. Trolier-McKinstry, P. C. McIntyre, Ann. Rev. Mater. Sci. 2000,30, 263

[3] C. H. Ahn, K. M. Rabe, J.-M. Triscone, Science 2004, 303, 488.

[4] E. Y. Tsymbal, H. Kohlstedt, Science 2006, 313, 181.

[5] M. Y. Zhuravlev, R. F. Sabirianov, S. S. Jaswal, E. Y. Tsymbal, Phys. Rev. Lett. 2005, 94, 246802.

[6] Kohlstedt, N. A. Pertsev, J. Rodríguez Contreras, R. Waser, Phys. Rev. B 2005, 72, 125341.

[7] V. Garcia, S. Fusil, K. Bouzehouane, S. Enouz-Vedrenne, N. D. Mathur, A. Barthelemy, M. Bibes, Nature 2009, 460, 81.

[8] A. Gruverman, D. Wu, H. Lu, Y. Wang, H. W. Jang, C. M. Folkman, M. Y. Zhuravlev, D. Felker, M. Rzchowski, C.-B. Eom, E. Y. Tsymbal, Nano Lett. 2009, 9, 3539

[9] M. Y. Zhuravlev, S. S. Jaswal, E. Y. Tsymbal, R. F. Sabirianov, Appl. Phys. Lett. 2005, 87, 222114.

[10] J. P. Velev, C.-G. Duan, J. D. Burton, A. Smogunov, M. K. Niranjan E. Tosatti, S. S. Jaswal, E. Y. Tsymbal, Nano Lett. 2009, 9, 427.

[11] V. Garcia, M. Bibes, L. Bocher, S. Valencia, F. Kronast, A. Crassous, X. Moya, S. Enouz-Vedrenne, A. Gloter, D. Imhoff, C. Deranlot, N. D. Mathur, S. Fusil, K. Bouzehouane, A. Barthelemy, Science 2010, 327, 1106.

[12] J. Junquera, Ph. Ghosez, Nature 2003, 422, 506.

[13] C. Lichtensteiger, J. M. Triscone, J. Junquera, Ph. Ghosez, Phys. Rev. Lett. 2005, 94, 047603.

[14] D. D. Fong, A. M. Kolpak, J. A. Eastman, S. K. Streiffer, P. H. Fuoss, G. B. Stephenson, C. Thompson, D. M. Kim, K. J. Choi, C. B. Eom, I. Grinberg, A. M. Rappe, Phys. Rev. Lett. 2006, 96, 127601.

[15] S. K. Streiffer, J. A. Eastman, D. D. Fong, C. Thompson, A. Munkholm, M. V. RamanaMurty, O. Auciello, G. R. Bai, G. B. Stephenson, Phys. Rev. Lett. 2002, 89, 067601.

[16] D. D. Fong, G. B. Stephenson, S. K. Streiffer, J. A. Eastman, O. Auciello, P. H. Fuoss, C. Thompson, Science 2004, 304, 1650.

[17] B.-K. Lai, I. Ponomareva, I. I. Naumov, I. Kornev, H. Fu, L. Bellaiche, G. J. Salamo, Phys. Rev. Lett. 2006, 96, 137602.

[18] P. Aguado-Puente, J. Junquera, Phys. Rev. Lett. 2008, 100, 177601.

[19] V. Nagarajan, J. Junquera, J. Q. He, C. L. Jia, R. Waser, K. Lee, Y. K. Kim, S. Baik, T. Zhao, R. Ramesh, Ph. Ghosez, K. M. Rabe, J. Appl. Phys. 2006, 100, 051609.

[20] S. V. Kalinin, D. A. Bonnell, Phys. Rev. B 2001, 63, 125411.

[21] D. J. Kim, J. Y. Jo, Y. S. Kim, Y. J. Chang, J. S. Lee, Jong-Gul Yoon, T. K. Song, T. W. Noh, Phys. Rev. Lett. 2005, 95, 237602

[22] A. K. Tagantsev, G. Gerra, J. Appl. Phys. 2006, 100, 051607.

[23] C. G. Duan, R. F. Sabirianov, W. N. Mei, S. S. Jaswal, E. Y. Tsymbal, Nano Lett. 2006, 6, 483.

[24] M. Stengel, D. Vanderbilt, N. A. Spaldin, Nat. Mat. 2009, 8, 392.

[25] G. Gerra, A. K. Tagantsev, N. Setter, Phys. Rev. Lett. 2007, 98, 207601.

[26] C.-Q. Jin, J.-S. Zhou, J. B. Goodenough, Q. Q. Liu, J. G. Zhao, L. X. Yang, Y. Yu, R. C. Yu, T. Katsura, A. Shatskiy, E. Ito, Proc. Natl. Acad. Sci. USA 2008, 105, 7115.

[27] H. J. Chang, S. V. Kalinin, A. N. Morozovska, M. Huijben, Y.-H. Chu, P. Yu, R. Ramesh, E. A. Eliseev, G. S. Svechnikov, S. J. Pennycook, A. Y. Borisevich, Adv. Mat. 2011, 23, 2474.

[28] R. Kretschmer, K. Binder, Phys. Rev. B 1979, 20, 1065. 
[29] X. Liu, Y. Wang, P. V. Lukashev, J. D. Burton, E. Y. Tsymbal, The interface dipole effect on thin-film ferroelectric stability: First-principles and phenomenological modeling, submitted.

[30] K. J. Choi, M. Biegalski, Y. L. Li, A. Sharan, J. Schubert, R. Uecker, P. Reiche, Y. B. Chen, X. Q. Pan, V. Gopalan, L.-Q. Chen, D. G. Schlom, C. B. Eom, Science 2004, 306, 1005.

[31] C. B. Eom, R. J. Cava, R. M. Fleming, J. M. Phillips, R. B. van Dover, J. H. Marshall, J. W. P. Hsu, J. J. Krajewski, W. F. Peck Jr., Science 1992, 258, 1766.

[32] D. H. Chang Y. S. Yoon, S. J. Kang, J. Korean Phys. Soc. 2001, 38, 277.
[33] A. F. Devonshire, Philos. Mag. 1951, 42, 1065

[34] S. V. Kalinin, B. J. Rodriguez, S.-H. Kim, S.-K. Hong, A. Gruverman, E. A. Eliseev, Appl. Phys. Lett. 2008, 92, 152906.

[35] D. A. Tenne, P. Turner, J. D. Schmidt, M. Biegalski, Y. L. Li, L. Q. Chen, A. Soukiassian, S. Trolier-McKinstry, D. G. Schlom, X. X. Xi, D. D. Fong, P. H. Fuoss, J. A. Eastman, G. B. Stephenson, C. Thompson, S. K. Streiffer, Phys. Rev Lett. 2009, 103, 177601.

[36] No sign of the EFM signal decay over the period of several hours has been detected suggesting high stability of the polarization states. 